\title{
Nonthermal production of gravitinos and inflatinos in the Inflationary Universe
}

\author{
Hans Peter Nilles and Marco Peloso
}

Physikalisches Institut, Universität Bonn, Nussallee 12, D-53115 Bonn, Germany

\begin{abstract}
The success of primordial nucleosynthesis imposes stringent bounds on the abundance of gravitational relics. This is particularly true for gravitinos, which - for models with gravitationally mediated supersymmetry breaking - are expected to have a mass below the $\mathrm{TeV}$ scale and thus to decay only after nucleosynthesis has concluded. We discuss the nonthermal production of gravitinos in models with several chiral fields, to be able to distinguish the mechanisms of supersymmetry breaking and inflation. Our explicit calculations show that the superpartner of the inflaton can be significantly generated. Gravitinos can be produced both at the preheating stage and at the subsequent inflaton/inflatino decay. We verify that this production is well below the nucleosynthesis bound, provided the sector responsible for the present supersymmetry breakdown is weakly (for example, only gravitationally) coupled to the one which drove inflation.
\end{abstract}

\section{Introduction}

There are several issues in astrophysics and cosmology where very high energy scales seem to be involved. For instance, consider the observation of cosmic rays with energy above the Greisen-Zatsepin-Kuzmin cut-off, or baryogenesis schemes as leptogenesis and GUT baryogenesis. The former may be explained with the decay of very massive particles with a lifetime comparable or larger than the present age of the Universe; the latter involve very massive right handed neutrinos or GUT bosons, whose out-of equilibrium decay generates the tiny baryonic asymmetry suggested by primordial nucleosynthesis. In most cases, these heavy particles have masses higher than the one of the inflaton, which typically ranges in the interval $\left(10^{10}-10^{13}\right) \mathrm{GeV}$ in the simplest models of "new" and "chaotic" inflation. As a consequence, reheating based on a perturbative decay of the inflaton can hardly account for their generation. Fortunately, the last decade has witnessed a drastic change in the theory of reheating, with the realization that nonperturbative effects can play a very significant role. The nonperturbative decay of the inflaton (which is due to the coherency of its oscillations) is typically a very quick phenomenon, followed by a phase of ordinary reheating or thermalization. For this reason, it is commonly denoted as preheating. 
One of the main successes of inflation is the generation of a nearly scale invariant spectrum of primordial metric fluctuations, as indicated by Cosmic Microwave Background and Large Scale Structure measurements. While the nearly scale invariance is a natural outcome of the slow roll of the inflaton field, the observed smallness of perturbations typically requires an ad hoc fine tuning of some parameter of the inflationary sector. As an example, in the two most standard models of single field chaotic inflation, $V(\phi)=m_{\phi}^{2} \phi^{2} / 2$ and $V(\phi)=\lambda \phi^{4} / 4$, one has to fix $m_{\phi}^{2} \sim 10^{-11} M_{P}^{2}$ for a massive inflaton $\left(M_{P}\right.$ here denotes the reduced Planck mass) or $\lambda \sim 10^{-13}$ in the massless inflaton case. Supersymmetry may provide a natural framework for the protection of such small couplings, and indeed inflationary supersymmetric models have been widely discussed in the literature. Since in chaotic and new inflationary schemes the inflaton field acquires values comparable or larger than $M_{P}$, complete models should include also supergravity effects rather than just global supersymmetry. These effects are mostly relevant at very early times, but one has to check that they are consistent with the phenomenology of the later evolution of the Universe. The most known example of possible difficulties in this direction is certainly provided by the gravitino problem.

The gravitino is necessary present in supergravity, since it is the supersymmetric partner of the graviton. It has four physical degrees of freedom, two "transverse" and "two" longitudinal. Actually, the longitudinal components are present only for broken supersymmetry, and they are provided by the goldstino field, which is a linear combination of the partners of the scalars (or gauge bosons) responsible for the supersymmetry breakdown. This mechanism is the "fermionic version" of spontaneous breaking of gauge symmetries, and indeed it is known as superhiggs mechanism. If supersymmetry has to solve the hierarchy problem, the scale of its breaking, and thus the mass of the gravitino (we restrict here to models of gravitational mediated supersymmetry breaking) cannot be too far from the electroweak scale, $m_{3 / 2} \sim 100 \mathrm{GeV}-1 \mathrm{TeV}$. If not the lightest supersymmetric particle, a gravitino with a mass in this range will decay only after nucleosynthesis has ended. The decay typically produces an electromagnetic shower, which can alter the successful predictions of standard primordial nucleosynthesis. Indeed, not to conflict with observations, the very strong limit

$$
Y_{3 / 2} \equiv \frac{n_{3 / 2}}{s} \lesssim 10^{-13}
$$

has to be imposed on the gravitino abundance (in standard notation, here $n_{3 / 2}$ denotes the gravitino number density, while $s$ the entropy density).

In the last twenty years, detailed calculations have been performed on the perturbative production of gravitinos after the inflaton decay products have thermalized. One finds that, for a gravitino with mass in the above range, a rather strong bound on the reheating temperature,

$$
T_{\text {rh }} \lesssim\left(10^{9}-10^{10}\right) \mathrm{GeV},
$$

has to be imposed in order to avoid excessive production. More recently, it has been wondered if preheating can also lead to a too large creation of gravitinos. This issue is, however, more complicated. Quite generally, preheating effects lead to the non-perturbative production of the fermionic partner of the inflaton, the inflatino, and of any other fermion which is strongly coupled to the inflaton field. While one easily finds that the transverse 
gravitino component is only weakly (i.e. gravitationally) coupled to the inflaton, and that its quanta are hence produced in a negligible amount, the production of the longitudinal component is very model dependent. If there is substantial mixing between the inflatino and the longitudinal component of the gravitino, the goldstino, preheating may result in an overproduction of gravitinos. During inflation and the beginning of reheating, supersymmetry is mainly broken by the inflaton implying a strong correspondence between the inflatino and goldstino at this early stage. However, this correspondence does not necessarily hold at late times, since supersymmetry may be broken by other fields in the true vacuum of the theory. Indeed, this is probably the most typical situation, as it is natural to distinguish between inflation and supersymmetry breaking due to the very different energy scales associated with the two phenomena. In this case, the final gravitino abundance can be much smaller than the inflatino one.

More accurately, the relic abundance of gravitinos will ultimately be related to the strength of the coupling between the inflationary sector and the one responsible for the present supersymmetry breakdown. The simplest possibility is to consider a model with two fields coupled only gravitationally, which is a simple prototype of more realistic schemes of gravitational mediation of supersymmetry breaking in a hidden sector. Even in this simplified model, the calculation of nonthermal gravitino production requires substantial work. First, one has to develop a new formalism, to be able to clearly define and compute the production in systems with several coupled fields. Subsequently, an extended numerical investigation has to be carried out to obtain reliable results. This calculation, which we have described in details in the works [1,2], is here summarized in section 2.

In accordance with the above discussion, the final gravitino production in these schemes turns out to be completely negligible also for the longitudinal component. The nonthermal production is much more relevant for the fermionic partner of the inflaton, the inflatino, which in some cases can be produced with a significant abundance. Thus, gravitinos may be overproduced through inflatino decay, if the channel inflatino $\rightarrow$ inflaton (or its scalar partner $)+$ gravitino is kinematically allowed. Depending on the relative masses of the inflaton and inflatino, significant production may be expected instead by the inverse process. This production may be particularly significant if the inflaton sector is coupled only gravitationally to matter, since in this case the above decays will have a rate comparable to the one generating the thermal bath. Indeed, in such a scenario, the decay channels into gravitinos need to be strongly suppressed. In the work [3] we have discussed the possible kinematic suppression of this channel, which translates into a rather strong lower bound on the scale of inflation. We have shown that this bound is satisfied by the simple single scale (supergravity) inflationary models. This analysis is summarized in section 3.

An extensive list of relevant bibliography can be found in $[1,2,3]$.

\section{Gravitino production at preheating}

The system we are considering has the matter content of two superfields $\Phi$ and $S$, with superpotential

$$
W=\frac{m_{\phi}}{2} \Phi^{2}+\mu^{2}(\beta+S)
$$


and with minimal Kähler potential $G=\mathcal{K}+\ln |W|^{2} \quad, \quad \mathcal{K}=\Phi^{\dagger} \Phi+S^{\dagger} S$. The field $\phi$ (that is, the scalar component of $\Phi$ ) acts as the inflaton. In the present model, supergravity corrections spoil the flatness of the potential during inflation, so that additional contributions must be relevant during inflation. However, we are interested in the dynamics of the system after inflation, when $\langle\phi\rangle \lesssim M_{p}$, and supergravity corrections are not important. We then assume that the superpotential (3) is valid at this stage, but we nonetheless normalize $m_{\phi} \sim 10^{13} \mathrm{GeV}$, as required by the COBE normalization of the CMB fluctuations for the "usual" chaotic inflation. A model where also new inflation is obtained was considered in [3], and it gives the same qualitative results as the one here described.

The superfield $S$ leads to the breaking of supersymmetry in the true vacuum owing to its "Polonyi" superpotential. By imposing $\beta=(2-\sqrt{3}) M_{p}$, one can indeed break supersymmetry while retaining a vanishing cosmological constant in the true vacuum, where the fields $s$ (the scalar component of the superfield $S$ ) has expectation value of the order of $M_{p}$. The gravitino mass in the vacuum is of the order $\mu^{2} / M_{p}$. In order to have a gravitino mass of about $100 \mathrm{GeV}$ (that is the expected value for the gravitino mass in gravity-mediated supersymmetry breaking models), $\mu \sim 10^{10} \mathrm{GeV}$ is required.

Right after inflation the field $\phi$ is oscillating about the bottom of its potential with frequency proportional to $m_{\phi}$. The time dependent expectation value of $\phi$ acts as an effective mass for the Polonyi scalar, which has vanishing expectation value at this stage. In this initial period the (time dependent) expectation value of $\phi$ is the main source of supersymmetry breaking. The amplitude of the oscillations of the field $\phi$ eventually decreases, due to the expansion of the Universe, and for times of the order of $m_{3 / 2}^{-1}$ the Polonyi scalar starts rolling down towards its true minimum and then oscillates about it. ${ }^{1}$

The system is thus governed by two time scales. At "early" times, of the order of $m_{\phi}^{-1}$, the only relevant dynamics is the one of the inflaton sector, that is also the main source of supersymmetry breaking. At "late" times, much larger than $m_{3 / 2}^{-1}$, the system behaves as if it was in its true vacuum, and supersymmetry is broken by the Polonyi sector. To be more specific, we define the dimensionless parameter $\hat{\mu}^{2} \equiv \mu^{2} /\left(m_{\phi} M_{p}\right) \sim m_{3 / 2} / m_{\phi}$, that gives the ratio of the two time scales in the system. If supersymmetry is supposed to solve the hierarchy problem, $\hat{\mu}^{2}$ should be of the order of $10^{-11}$. Such a small parameter implies a very large difference between the two time scales of the problem, which is a source of technical difficulties in the numerical computations. As a consequence, we could not study the evolution of the system for such a small value of $\hat{\mu}^{2}$. Thus, we kept it as a free parameter and we studied how a variation of $\hat{\mu}^{2}$ affects the scaling of the relevant quantities.

During the evolution, both the kinetic and potential energies of the two fields contribute to the supersymmetry breaking. One can define

$$
f_{\phi_{i}}^{2} \equiv m_{i}^{2}+\frac{1}{2}\left(\frac{d \phi_{i}}{d t}\right)^{2}
$$

with $m_{i}=\exp \left(\mathcal{K} M_{p}^{-2} / 2\right)\left[\partial_{i} W+M_{p}^{-2} \partial_{i} \mathcal{K} W\right]$. The quantities $f_{i}$ give a "measure"

\footnotetext{
${ }^{1}$ We neglect here the Polonyi problem associated with the late times oscillations of $S$.
} 
of the size of the supersymmetry breaking provided by the $F$ term associated with the $i$-th scalar field. More precisely, we are interested in the normalized quantities $r_{i} \equiv$ $f_{i}^{2} /\left(f_{1}^{2}+f_{2}^{2}\right)$, which indicate the relative contribution of the two scalars. It can be easily verified [1] that in the initial stages $r_{\phi} \simeq 1$, while $r_{s} \simeq 1$ at late times. The regime of equal contribution is around $t m_{\phi}=\hat{\mu}^{-2}$.

Let us now consider the fermionic content. We denote the fermions of the two chiral multiplets by $\tilde{\phi}$ (the "inflatino") and $\tilde{s}$ (the "Polonyino"). One linear combination of them is the goldstino $v$, while the one orthogonal to $v$ is denoted by $\Upsilon$. Initially, $v \equiv \tilde{\phi}$, while $v \equiv \tilde{s}$ at late times. In addition, we have the gravitino field, whose longitudinal and transverse component are denoted by $\theta$ and by $\psi_{i}^{T}$, respectively. The transverse component is decoupled from the other fermion fields, and its quanta are produced only gravitationally. The longitudinal gravitino component, which in the super-higgs mechanism is provided by the goldstino $v$, is however coupled with $\Upsilon$. The computation of the occupation numbers of the fermions $\theta$ and $\Upsilon$ is far from trivial and requires a significant extension of the existing formalism for nonperturbative production in the one field case. The details of the calculation are reported in [2].

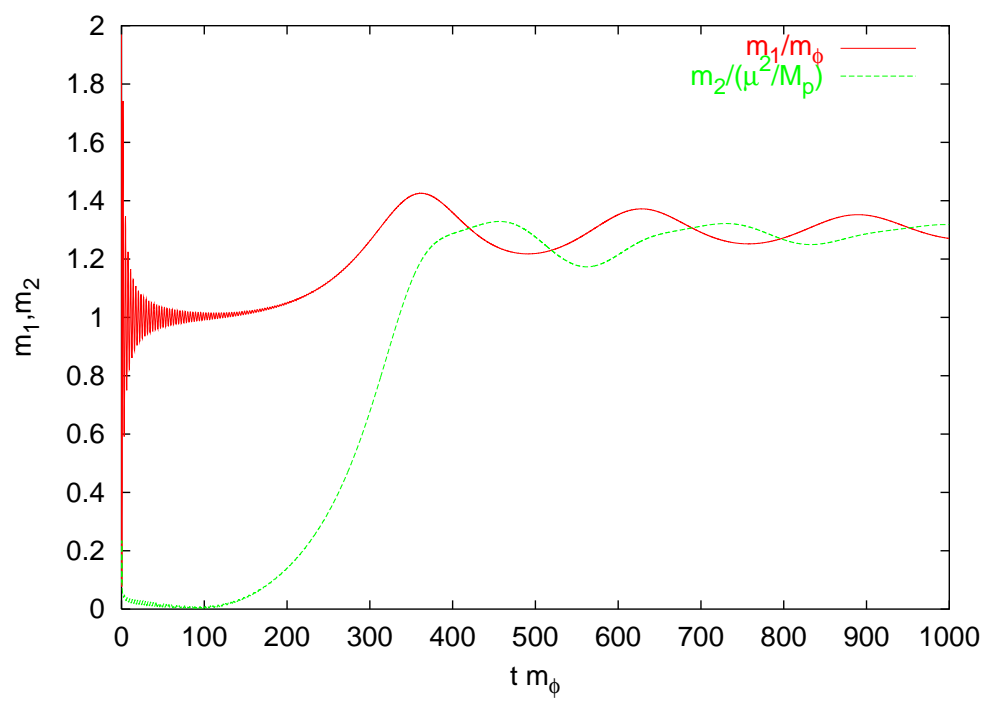

Figure 1: Evolution of the masses of the two fermionic eigenstates. For illustrative porpoises we take $\hat{\mu}^{2}=10^{-2}$. Notice the different normalizations for the two masses.

As a starting point, one has to diagonalize (at each time) the coupled $\theta-\Upsilon$ system. We denote the two fermionic mass eigenstates by $\psi_{1}$ and $\psi_{2}$. In fig. 1 we show ${ }^{2}$ the evolution of their masses for the specific case $\hat{\mu}^{2}=10^{-2}$. The most relevant information which can be extracted by this evolution is very clear: at late times the fields $\psi_{1}$ and $\psi_{2}$ have, respectively, the mass of the inflatino and of the gravitino field. That is, at late times we have the identification $\psi_{1} \equiv \tilde{\phi} \equiv \Upsilon, \psi_{2} \equiv \theta \quad(\tilde{s}=v=0$, being the goldstino). This situation is orthogonal to the initial one, when $\psi_{1} \equiv \theta, \psi_{2} \equiv \tilde{s} \equiv \Upsilon$ $(\tilde{\phi}=v=0)$. At intermediate times, when supersymmetry is broken by both scalar fields,

\footnotetext{
${ }^{2}$ From fig. 1 , one may be tempted to identify $\psi_{1} \equiv \tilde{\phi}$ and $\psi_{2} \equiv \tilde{s}$. Although this identification is rigorous only at the beginning and at the end of the evolution, it can be used for an "intuitive" understanding of the system.
} 
the gravitino is a mixture of $\psi_{1}$ and $\psi_{2}$. To qualitatively appreciate the evolution of the gravitino occupation number, we may consider $N_{\theta} \equiv r_{1} N_{1}+r_{2} N_{2}$ and the orthogonal combination $N_{\Upsilon} \equiv r_{2} N_{1}+r_{1} N_{2}$, where $r_{i}$ are the relative contributions of the two scalars to supersymmetry breaking defined above.

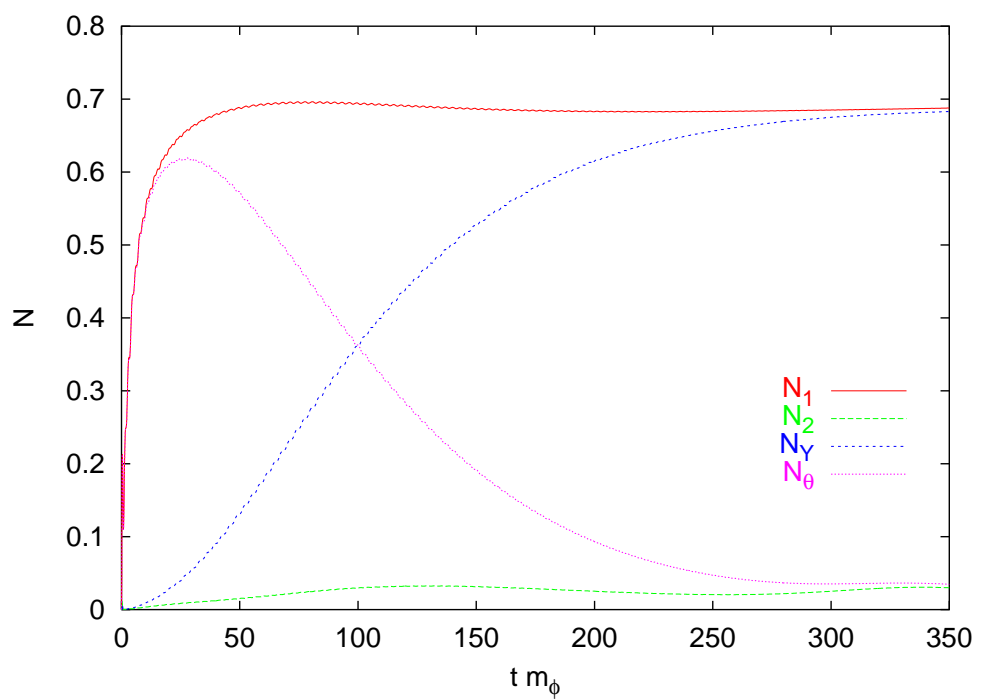

Figure 2: Evolution of $N_{\theta}$ and $N_{\Upsilon}$ for $\hat{\mu}^{2}=10^{-2}$ and $k=m_{\phi}$. See the text for details.

We show in fig. 2 the evolution of $N_{1}, N_{2}, N_{\theta}, N_{\Upsilon}$ for modes of comoving momentum $k=m_{\phi}$ (the scale factor of the Universe is normalized to one at the end of inflation) and for $\hat{\mu}^{2}=10^{-2}$. Notice that (by construction) $N_{\theta} \equiv N_{1}$ at early times, while $N_{\theta} \equiv N_{2}$ at late ones. We also see that $\psi_{1}$ is populated on time scales $m_{\phi}^{-1}$, while $\psi_{2}$ on time scales $\hat{\mu}^{-2} m_{\phi}^{-1}$. This feature is common for all $\hat{\mu}^{2}[2]$. We remark that the identification $\theta \equiv r_{1} \psi_{1}+r_{2} \psi_{2}$ should be taken only as a qualitative indication. However, the most relevant identification $\theta \equiv \psi_{2}$ at late times is a rigorous one, as should be clear from the above discussion.

We are now ready to present our most important result: the occupation number of the two fermionic mass eigenstates at the end of the process. As we have said, the realistic case $\hat{\mu}^{2}=10^{-11}$ is far from our available resources, so we kept $\hat{\mu}^{2}$ as a free parameter and performed numerical calculations down to $\hat{\mu}^{2}=10^{-6}$. The results for $\hat{\mu}^{2}=10^{-11}$ can be clearly extrapolated from the ones we are going to present. Moreover, the case $\hat{\mu}^{2}=0$ can be studied analytically [2], and it agrees with the limit $\hat{\mu} \rightarrow 0$ deduced from the numerical results. For $\hat{\mu}=0$, only the inflatino is produced. The mass of the Polonyi fermion does instead vanish identically, so no quanta of this particle are produced at preheating [2]. Notice that $\hat{\mu}=0$ corresponds to a situation with unbroken susy in the vacuum, and it reproduces the models with one single field studied so far. We stress that in this case only the inflatino is produced at preheating.

As we showed in $[1,2]$, the field $\psi_{1}$ has occupation number of order one up to $k \sim m_{\phi}$, and then rapidly decreases as $k^{-4}$. The final spectrum is practically independent of the value of $\hat{\mu}^{-2}$. These features are easily explained: the eigenstate $\psi_{1}$ is associated to the inflatino, that is produced by the coherent oscillations of the inflaton; the inflaton dynamics occurs on time scales of the order of $m_{\phi}^{-1}$, and is independent of $\hat{\mu}^{2}$. 


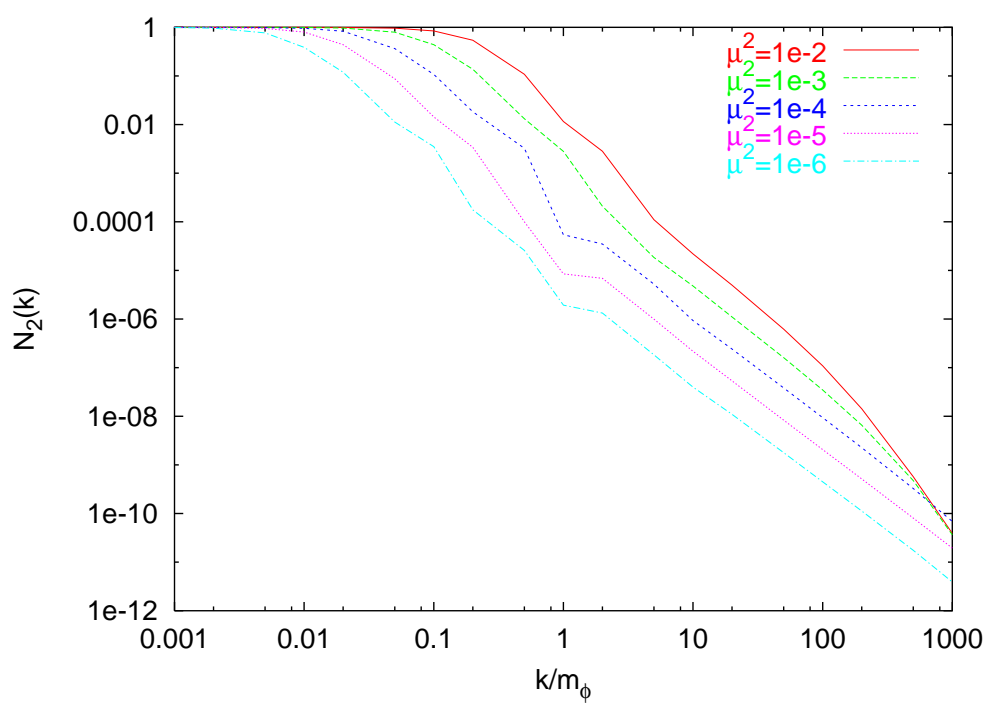

Figure 3: Spectrum of gravitinos at late times.

The spectrum of $\psi_{2}$ - the late time gravitino - is shown in fig. 3 for different values of $\hat{\mu}^{2},{ }^{3}$ and exhibits instead a more significant dependence on $\hat{\mu}^{2}$. One finds that these quanta are mainly produced during the first oscillations of the Polonyi field, with a typical physical momentum of the order the Polonyi $\left(\sim m_{3 / 2}\right)$ mass $\mu^{2} / M_{P}$. The total number of particles produced in this case is thus an increasing function of $\hat{\mu}^{2}$. One easily realizes that the production is expected to be completely negligible in the physically relevant case $\hat{\mu}^{2}=10^{-11}$.

\section{Gravitino production through the inflaton/inflatino decay}

In this section we generalize the results discussed above, considering also gravitino production from the decay of inflaton and inflatino. Since we will always consider a supersymmetry breaking scale much smaller than the scale of inflation, it is worth noticing that the decays of the inflaton and the inflatino are expected to occur nearly simultaneously. As we have said, the potential of the inflaton field during inflation is constrained by the magnitude of density fluctuations following from CMB temperature measurements. In the slow roll regime, one has to impose

$$
V_{60}^{1 / 4} \simeq 0.027 \epsilon_{60}^{1 / 4} M_{P}
$$

where $\epsilon^{2} \equiv M_{p}^{2}\left(V^{\prime} / V\right)^{2} / 2 \ll 1$ is one of the two slow-roll parameters (prime denoting derivative with respect to $\phi$ ) and the suffix 60 reminds us that the two quantities have to be evaluated when the scales measured by COBE left the horizon, about 60 e-foldings

\footnotetext{
${ }^{3}$ These spectra are shown at the time $t=10 \hat{\mu}^{-2} m_{\phi}^{-1}$. In the $\hat{\mu}=10^{-2}-10^{-4}$ cases we have continued the evolution further, until the spectra stop evolving. In these cases, we have found that the spectra very slightly decrease for $t>10 \hat{\mu}^{-2} m_{\phi}^{-1}$. Thus, the results shown in fig. 3 give an accurate upper bound on the final gravitino abundance.
} 
before the end of inflation. As stated in the introduction, this relation uniquely fixes the scale of the potential in the simplest models of one field inflation and potential $V \propto \phi^{n}$. However, models with a much smaller scale than the one reported there and acceptable density fluctuations can be constructed. As follows from eq. (5), in models with one single field this can be done at the expense of a small $\epsilon$ parameter, that is by taking a very flat potential during inflation. Such a flat potential may arise more naturally if more scalar fields are present, as for example in hybrid inflationary models.

Due to this freedom, in this section we discuss the production of gravitinos with a generic inflationary scale. To be precise, we denote by $\Delta^{4}$ the value of the inflaton potential at the end of inflation, when the reheating stage begins. Due to the slow motion of $\phi$ during inflation, this scale is typically very close to $V_{60}$. During reheating, the inflaton field oscillates about the minimum $\phi_{0}$ of $V$, with $V\left(\phi_{0}\right)=0$. As is typical for a massive inflaton, we assume that the quadratic term dominates the Taylor expansion of $V$ around $\phi_{0}$. We denote by $F$ the amplitude of the inflaton oscillations at the initial time $t \sim H^{-1} \sim M_{P} / \Delta^{2}$. In the present discussion we keep also the value of $F$ as a free parameter. As we will now show, the number density of produced gravitinos is more closely related to the inflaton mass $m_{\phi}$ rather than to the scale $\Delta$. For a quadratic potential and generic values of $\Delta$ and $F$, one has $m_{\phi} \simeq \Delta^{2} / F$. Note that the model considered in the previous section is characterized by $F \simeq M_{P}$ and $m_{\phi} \simeq 10^{13} \mathrm{GeV}$.

We discuss here the case in which the inflaton decays only gravitationally. A more general analysis where also nongravitational inflaton decays are considered was performed in [3]. The gravitational decay rate of the inflaton is given by $\Gamma \simeq m_{\phi}^{3} / M_{p}^{2}$, leading to the reheating temperature $T_{\mathrm{rh}} \simeq\left(\Gamma M_{P}\right)^{1 / 2} \simeq \sqrt{m_{\phi}^{3} / M_{P}}$. The thermal bound (2) thus simply gives

$$
m_{\phi} \lesssim 10^{12} \mathrm{GeV}
$$

The inflaton number density at its decay can be estimated to be $n_{\phi}=V(\phi) / m_{\phi}$ at $t \equiv \tau_{\phi}=\Gamma^{-1}$. The inflaton "abundance" at the decay time is thus approximatively given by

$$
Y_{\phi} \simeq \frac{n_{\phi}}{\rho_{\phi}^{3 / 4}} \simeq \frac{T_{R}}{m_{\phi}} \simeq \sqrt{\frac{m_{\phi}}{M_{P}}}
$$

The abundance of inflatinos produced nonthermally by the inflaton oscillations is also easily evaluated, by remembering that inflatinos are produced at preheating up to physical momentum $m_{\phi}$, and with a physical number density of the order $n_{\tilde{\phi}} \simeq 10^{-2} m_{\phi}^{3}$ $\times\left[a\left(t=m_{\phi}^{-1}\right) / a(t)\right]^{3}$. The inflatino abundance at $t=\tau_{\phi}$ is thus

$$
Y_{\tilde{\phi}} \simeq \frac{n_{\tilde{\phi}}}{\rho_{\phi}^{3 / 4}} \simeq 10^{-2} \frac{m_{\phi} T_{R}}{M_{P}^{2}} \simeq 10^{-2}\left(\frac{m_{\phi}}{M_{P}}\right)^{5 / 2}
$$

Also the abundance of gravitinos produced nonthermally becomes smaller as $m_{\phi}$ decreases, so that it is always negligible. Indeed, gravitinos will still be mainly produced at the time $t \sim m_{3 / 2}^{-1}$ with a typical momentum $k \sim m_{3 / 2}^{-1}$, independent of the value of $m_{\phi}$. However, a lighter inflaton implies a longer lifetime $\tau_{\phi}$. Since the quantity $n_{3 / 2} / \rho_{\phi}^{3 / 4}$ decreases in the time interval $m_{3 / 2}<t<\tau_{\phi}$, lowering $m_{\phi}$ will thus decrease the final nonthermal gravitino abundance. 
We see that the inflaton abundance is always higher than the one of inflatinos. Indeed, once the bound (6) is respected, the inflatino abundance is smaller than the limit $10^{-13}$ reported in equation (1). Even if all the inflatino quanta produce a gravitino when they decay, this would not lead to an overproduction of gravitinos (the inflatino abundance can be higher for a massless inflaton, e. g. with potential $V(\phi) \propto \phi^{n}, n>2$; see $[2,3]$ for details). Gravitino production can instead be significant through the $\phi \rightarrow \tilde{\phi} \tilde{G}$ decay, if kinematically allowed.

Indeed, assuming that the branching fraction for this decay is $1 / N$, from eq. (7) we get the gravitino abundance $Y_{3 / 2}=Y_{\phi} / N=\sqrt{m_{\phi} / M_{P}} / N$. If we now take $N \sim 100$ as suggested by the degrees of freedom of the Minimal Supersymmetric Standard Model, the bound (1) requires $m_{\phi} \lesssim 10^{9} \mathrm{GeV}$. We see that this constraint is much stronger then the one coming from the thermal production (6). Of course, if it happens that the decay inflaton $\rightarrow$ inflatino + gravitino is kinematically forbidden $\left(\left|m_{\phi}-m_{\tilde{\phi}}\right|<m_{3 / 2}\right)$, then this last bound does not hold (this occurs for example for the specific choice of a present supersymmetry breaking provided by a Polonyi superpotential [3]). In addition, if the scale of supersymmetry breaking is significantly below that of inflation, i.e., $\mu \ll \Delta$, then even though the decay $\phi \rightarrow \tilde{\phi}+\tilde{G}$ is allowed, it will be naturally kinematically suppressed with respect to the other decay channels of the inflaton field. This will open an allowed window for $m_{\phi}$ even in the simplest scenarios. Independent of the details of the decay, the rate will always carry a final state momentum suppression factor. The overall decay rate can be written as $\Gamma \sim\left(1 / m_{\phi}\right)|\mathcal{M}|^{2}\left(p / m_{\phi}\right)$, where $|\mathcal{M}|$ is the amplitude for decay and the final state momentum suppression factor is

$$
2 p / m_{\phi}=\left(1-\frac{2\left(m_{\tilde{\phi}}^{2}+m_{3 / 2}^{2}\right)}{m_{\phi}^{2}}+\frac{\left(m_{\tilde{\phi}}^{2}-m_{3 / 2}^{2}\right)^{2}}{m_{\phi}^{4}}\right)^{1 / 2} \sim \frac{m_{3 / 2}}{m_{\phi}}
$$

Thus in models in which $m_{3 / 2} \ll m_{\phi}$, there will be a significant suppression in the production of gravitinos by either inflaton or inflatino decay (note that additional suppression may come from the specific form of the amplitude $\mathcal{M}$ as well). Taking into account the suppression factor (9) in the bound for the gravitino production by inflaton decay, and combining it with the limit coming from the thermal production, we find that the inflaton mass has to lay within the interval [3]

$$
10^{8} \mathrm{GeV}\left(\frac{m_{3 / 2}}{100 \mathrm{GeV}}\right)^{2} \lesssim m_{\phi} \lesssim 10^{12} \mathrm{GeV} .
$$

Note that the upper bound on $m_{\phi}$ comes from avoiding a too quick inflaton decay, with a consequent too high reheating temperature. The lower bound is instead due to the fact that for too low inflaton mass the kinematical suppression factor (9) is no longer capable of maintaining a safe small branching ratio for the $\phi \rightarrow \tilde{\phi}+\tilde{G}$ channel. The simplest models of single scale inflation with purely gravitational decays and a scale set by (5), have typically $F \simeq M_{P}$ and $\Delta \simeq$ few $\times 10^{-4} M_{p}$. In this case the gravitino mass is slightly higher than $10^{10} \mathrm{GeV}$, well within the allowed range (10). We thus conclude that these models do not suffer from a gravitino problem. 


\section{Acknowledgments}

Work supported in part by the European Community's Human Potential Programme under contracts HPRN-CT-2000-00131 Quantum Spacetime, HPRN-CT-2000-00148 Physics Across the Present Energy Frontier and HPRN-CT-2000-00152 Supersymmetry and the Early Universe.

\section{References}

[1] H. P. Nilles, M. Peloso, and L. Sorbo, Phys. Rev. Lett. 87, 051302 (2001).

[2] H. P. Nilles, M. Peloso, and L. Sorbo, JHEP 0104, 004 (2001).

[3] H. P. Nilles, K. A. Olive, and M. Peloso, Phys.Lett. B522, 304 (2001). 